DEPARTMENT OF ECONOMICS

Working Paper Series

Pure Strategy Equilibria in Symmetric Two-Player Zero-Sum Games

Peter Duersch

University of Heidelberg

Joerg Oechssler

University of Heidelberg

Burkhard Schipper

University of California Davis

November 23, 2010

Paper \# 10-21

We observe that a symmetric two-player zero-sum game has a pure strategy equilibrium if and only if it is not a generalized rock-paper-scissors matrix. Moreover, we show that every finite symmetric quasiconcave two-player zero-sum game has a pure equilibrium. Further sufficient conditions for existence are provided. Our findings extend to general two-player zero-sum games using the symmetrization of zero-sum games due to von Neumann. We point out that the class of symmetric two-player zero-sum games coincides with the class of relative payoff games associated with symmetric two-player games. This allows us to derive results on the existence of finite population evolutionary stable strategies.

Department of Economics

One Shields Avenue

Davis, CA 95616

(530)752-0741

http://www.econ.ucdavis.edu/working_search.cfm 


\title{
Pure Strategy Equilibria in Symmetric Two-Player Zero-Sum Games*
}

\author{
Peter Duersch $^{\dagger} \quad$ Jörg Oechssler ${ }^{\ddagger} \quad$ Burkhard C. Schipper ${ }^{\S}$
}

November 22, 2010

\begin{abstract}
We show that a symmetric two-player zero-sum game has a pure strategy equilibrium if and only if it is not a generalized rock-paper-scissors matrix. Moreover, we show that every finite symmetric quasiconcave two-player zero-sum game has a pure equilibrium. Further sufficient conditions for existence are provided. We point out that the class of symmetric two-player zero-sum games coincides with the class of relative payoff games associated with symmetric two-player games. This allows us to derive results on the existence of finite population evolutionary stable strategies.
\end{abstract}

Keywords: Symmetric two-player games, zero-sum games, Rock-Paper-Scissors, single-peakedness, quasiconcavity, finite population evolutionary stable strategy, saddle point, exact potential games.

JEL-Classifications: C72, C73.

*An earlier working paper version has been circulated under the title "Pure Saddle Points and Symmetric Relative Payoff Games". Some of the material has previously been part of the paper "Unbeatable imitation" presented at the International Conference on Game Theory in Stony Brook, 2009. We thank Reinhard John, the associated editor and two anonymous referees for extremely helpful suggestions.

${ }^{\dagger}$ Department of Economics, University of Heidelberg, Email: peter.duersch@awi.uni-heidelberg.de

${ }^{\ddagger}$ Department of Economics, University of Heidelberg, Email: oechssler@uni-hd.de

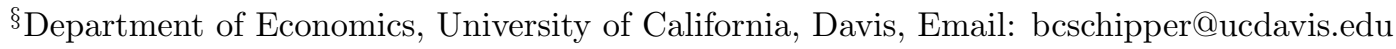




\section{Introduction}

Many zero-sum games do not have a solution without allowing for mixed strategies. What is the class of zero-sum games possessing pure equilibria? Some answers to these questions have been given by Shapley (1964) and Radzik (1991). For instance, Shapley (1964) showed that a finite two-player zero-sum game has a pure equilibrium if every $2 x 2$ submatrix of the game has a pure equilibrium. Radzik (1991) showed that a two-player zero-sum game whose columns are quasiconcave (i.e. single-peaked) and whose rows are quasiconvex has a pure equilibrium if and only if every submatrix "along the diagonal" has a pure equilibrium. Although both results apply to symmetric two-player zero-sum games, none of their results exploits the symmetry property.

In this paper we are interested in pure equilibria of symmetric two-player zero-sum games. It is well known that for instance the rock-paper-scissors game has no pure equilibrium. We show that this holds more generally. We say that a symmetric two-player zero-sum game is a generalized rock-paper-scissors matrix ( $g R P S)$ if for each column there exists a row with a strictly positive payoff. This notion allows us to characterize symmetric zero-sum games possessing pure equilibria. A symmetric two-player zero-sum game has a pure equilibrium if and only if it is not a gRPS. Moreover, we show that every finite symmetric quasiconcave two-player zero-sum game has a pure equilibrium. We also provide sufficient conditions for existence in terms of increasing and decreasing differences, potentials, and additive separability of payoffs. It turns out that symmetric two-player zero-sum games are a very special class of games in which increasing and decreasing differences, the existence of an exact potential, and additively separable payoffs coincide.

Symmetric two-person zero-sum games are often thought to be a very restricted class that is less relevant to economics. However, in Section 3 we shall argue that they arise naturally when relative payoffs of arbitrary symmetric two-player games are considered. ${ }^{1}$ The reason is simply that relative payoff functions give rise to zero-sum games by construction. Schaffer $(1988,1989)$ introduced the notion of finite population evolutionary stable strategies (fESS) and observed that a fESS of the original (arbitrary) symmetric game coincides with the Nash equilibrium of the (zero-sum) relative payoff game. ${ }^{2}$ Thus,

\footnotetext{
${ }^{1}$ There is some experimental evidence that players consider not only their absolute payoffs but also relative payoffs. Early experiments include Nydegger and Owen (1974) and Roth and Malouf (1979). More recently, relative payoff concerns have been studied in behavioral economics and experimental economics under the label of "inequity aversion".

${ }^{2}$ This relationship between Nash equilibrium and fESS has been analyzed for competitive games by
} 
when we apply our equilibrium existence results to the relative payoff game, we also obtain existence results for fESS of the underlying original game. This way, our results can be applied for example to Cournot duopoly, Bertrand duopoly, public goods games, common pool resource games, minimum effort coordination games, synergistic relationships, arms race, Diamond's search, Nash demand game, or rent seeking. We also show that a symmetric two-player game is an exact potential game if and only if its relative payoff game is an exact potential game. This is useful because the existence of an exact potential of a symmetric zero-sum game is easy to verify.

The fESS of a game is of relevance when evolution operates in a finite (playing-thefield) population. It is also important because frequently it coincides with the stochastically stable states of imitate-the-best dynamics. ${ }^{3}$ Thus, when players imitate in such games, we should expect the outcome to be a fESS. The results developed here are also used in our companion paper, Duersch, Oechssler, and Schipper (2010). There we characterize the class of games in which "imitate-the-best" can not be exploited by any other decision rule.

In the next section, we study the existence of pure equilibria in symmetric two-player zero-sum games. In Section 3 we apply our results to relative payoff games and the existence of finite population evolutionary stable strategies.

\section{Symmetric Zero-Sum Games}

We consider a symmetric two-player game $(X, \pi)$, in which both players are endowed with the same (finite or infinite) set of pure actions $X$. For each player, the bounded payoff function is denoted by $\pi: X \times X \longrightarrow \mathbb{R}$, where $\pi(x, y)$ denotes the payoff to the player choosing the first argument when his opponent chooses the second argument. In this section we will restrict attention to zero-sum games. Symmetry and the zerosum property together imply then that $\pi(x, y)=-\pi(y, x) .{ }^{4}$ Note that in a symmetric zero-sum game, the payoffs on the main "diagonal" must be zero.

Definition 1 In a symmetric two-player zero-sum game $(X, \pi)$, a pair of strategies $(x, y)$

Ania (2008) and for "weakly competitive" games by Hehenkamp et al. (2010).

${ }^{3}$ See e.g. Alós-Ferrer and Ania (2005), Hehenkamp, Leininger, and Possajennikov (2004), Leininger (2006), Matros, Temzelides, and Duffy (2009), Possajennikov (2003), Schipper (2003), Tanaka (2000), and Vega-Redondo (1997)

\footnotetext{
${ }^{4}$ The payoff matrix of symmetric zero-sum game is skew-symmetric.
} 
is a pure equilibrium if $\pi(x, y)=\max _{x^{\prime} \in X} \pi\left(x^{\prime}, y\right)=\min _{y^{\prime} \in X} \pi\left(x, y^{\prime}\right)$. A pure equilibrium $(x, y)$ is symmetric if $x=y$.

For obvious reasons, an equilibrium in two-player zero-sum games is often called a "saddle point". In a symmetric game, if $(x, y)$ is a pure equilibrium, so is $(y, x)$. By the rectangularity of equilibria in two-player zero-sum games, we also have that $(x, x)$ and $(y, y)$ are pure equilibria. This yields the following known fact (see e.g. Nash, 1951).

Remark 1 A symmetric two-player zero-sum game $(X, \pi)$ has a pure equilibrium if and only if it has a symmetric pure equilibrium.

A well known example for a symmetric zero-sum game without a pure equilibrium is the game "Rock-Paper-Scissors". ${ }^{5}$

$$
\begin{aligned}
& R \\
& P \\
& S
\end{aligned} \quad\left(\begin{array}{ccc}
R & P & S \\
0 & -1 & 1 \\
1 & 0 & -1 \\
-1 & 1 & 0
\end{array}\right)
$$

This example can be generalized to the following class of games.

Definition 2 (Generalized Rock-Paper-Scissors Matrix (gRPS)) A symmetric zerosum game $(X, \pi)$ is a generalized rock-paper-scissors matrix if in each column there exists a row with a strictly positive payoff to the row player i.e. if for all $y \in X$ there exists a $x \in X$ such that $\pi(x, y)>0 .^{6}$

This definition allows us to provide a full characterization of pure equilibria in symmetric two-player zero-sum games.

Theorem 1 A symmetric two-player zero-sum game $(X, \pi)$ possesses a pure equilibrium if and only if it is not a generalized rock-paper-scissors matrix.

Proof. If $(X, \pi)$ has no pure equilibrium (and in particular no pure symmetric equilibrium), then for all $y \in X$ there is $x \in X$ such that $\pi(x, y)>\pi(y, y)=0$. Thus, $(X, \pi)$ is gRPS. Conversely, if $(X, \pi)$ has a pure equilibrium, by Remark 1 it has a symmetric

\footnotetext{
${ }^{5}$ The game was already described by von Neumann (1928, p. 303).

${ }^{6}$ In the finite strategy case, an alternative way of characterizing a gRPS game would be to say that the game has a strictly positive minimax with respect to pure strategies i.e. if $\min _{y} \max _{x} \pi(x, y)>0$.
} 
equilibrium. Thus, there is $y \in X$ such that $\pi(x, y) \leq \pi(y, y)$ for all $x \in X$, which implies that $(X, \pi)$ is not $\mathrm{gRPS}^{7}$

A symmetric $2 \times 2$ zero-sum game cannot be a gRPS. If one of the row player's offdiagonal relative payoffs is $a>0$, then the other must be $-a$ violating the definition of gRPS. "Matching pennies" is not a counter-example because it is not symmetric. Thus we have the following corollary.

Corollary 1 Every symmetric 2x2 zero-sum game possesses a pure equilibrium.

In the following we shall study how several properties that are commonly assumed to hold for games relate to the gRPS property.

Definition 3 (Quasiconcave) A symmetric two-player game $(X, \pi)$ is quasiconcave (or single-peaked) if there exists a total order $<$ on $X$ such that for each $x, x^{\prime}, x^{\prime \prime}, y \in X$ and $x^{\prime}<x<x^{\prime \prime}$, we have that $\pi(x, y) \geq \min \left\{\pi\left(x^{\prime}, y\right), \pi\left(x^{\prime \prime}, y\right)\right\}$.

That is, a symmetric game is quasiconcave if each column has a single peak.

Theorem 2 Every finite quasiconcave symmetric two-player zero-sum game has a pure equilibrium.

The proof follows as a corollary from Theorem 1 and the following lemma.

Lemma 1 A finite quasiconcave symmetric two-player zero-sum game is not a gRPS.

Proof. Suppose by contradiction that the finite quasiconcave symmetric zero-sum game $(X, \pi)$ is a gRPS. Note first that if $\pi(\cdot, y)$ is quasiconcave in the first argument, i.e., if $x^{\prime}<x<x^{\prime \prime}$ implies that $\pi(x, y) \geq \min \left\{\pi\left(x^{\prime}, y\right), \pi\left(x^{\prime \prime}, y\right)\right\}$, then by symmetry, $\pi(y, x) \leq \max \left\{\pi\left(y, x^{\prime}\right), \pi\left(y, x^{\prime \prime}\right)\right\}$, i.e. $\pi(x, \cdot)$ is quasiconvex in the second argument.

Let $\left(x_{k}, x_{\ell}\right)$ be the left-most cell with a strictly positive entry that is above the main diagonal, i.e. $\pi\left(x_{k}, x_{\ell}\right)>0$, where $x_{\ell}:=\arg \min _{x^{\prime \prime}}\left\{\pi\left(x^{\prime}, x^{\prime \prime}\right)>0\right.$ and $\left.x^{\prime \prime}>x^{\prime}\right\}$ and $x_{k}:=$ $\arg \min _{x^{\prime}}\left\{\pi\left(x^{\prime}, x_{\ell}\right)\right\}$. If there are several such entries in column $x_{\ell}$, we choose without loss of generality the lowest one. Such an entry exists since $(X, \pi)$ is a gRPS and finite (i.e., the last column must have a strictly positive entry above the main diagonal).

${ }^{7}$ We thank the Associate Editor for suggesting this simple proof. 
By symmetry, $\left(x_{\ell}, x_{k}\right)$ is below the main diagonal and $\pi\left(x_{\ell}, x_{k}\right)<0$. By quasiconcavity, all entries in the column $x_{k}$ below $x_{\ell}$ are also negative, $\pi\left(x, x_{k}\right)<0$, for all $x>x_{\ell}$.

Since rows are quasiconvex, it follows that $\pi\left(x_{\ell}, x\right) \leq 0$ for all $x$ such that $x_{k}<x<x_{\ell}$. The same holds for all lower rows, $\pi\left(x^{\prime}, x\right) \leq 0$, for all $x^{\prime}>x_{\ell}, x_{k}<x<x^{\prime}$. This defines a "trapezoid" $\Pi_{n e g}$ of payoff entries below the diagonal that does not contain any strictly positive entries.

Now, look specifically at column $x_{\ell-1} \cdot \Pi_{n e g}$ contains all entries in this column that are below the diagonal. However, this column must have a positive entry since the game is a gRPS. Therefore, the column has to have a positive entry above the diagonal. But this is a contradiction to the fact that $\left(x_{k}, x_{\ell}\right)$ is the left-most cell with a positive entry above the main diagonal.

Note that if the finite zero-sum game is not symmetric but quasiconcave, then it does not need to have a pure equilibrium. A counter example is presented in Radzik (1991, p. 26). Hence, symmetry is crucial for the result.

The converse to Theorem 2 is not true as the following example shows.

Example 1 Consider the following "Rock-Paper-Scissors" game augmented by an additional strategy " $B$ ".

$$
\begin{aligned}
& R \\
& R \\
& P \\
& S \\
& B
\end{aligned} \quad\left(\begin{array}{cccc}
R & P & S & B \\
0 & -1 & 1 & -1 \\
1 & 0 & -1 & -1 \\
-1 & 1 & 0 & -1 \\
1 & 1 & 1 & 0
\end{array}\right)
$$

Clearly, it is not a $g R P S$ since for column " $B$ " there fails to exist a row yielding a strictly positive payoff. Thus, the game possesses a pure equilibrium, $(B, B)$. Yet, no matter how strategies are ordered, the game fails to be quasiconcave. Hence, there are symmetric two-player zero-sum games that are neither generalized rock-paper-scissors games nor quasiconcave.

Other "second-order" conditions are commonly explored in the literature when analyzing the existence of pure equilibria. We will consider increasing and decreasing differences, additive separability, and potentials. Surprising to us, it turns out that for symmetric two-player zero-sum games these conditions are all equivalent.

Definition 4 (Increasing and decreasing differences) Let $X$ be a totally ordered set. A payoff function $\pi$ has decreasing (resp. increasing) differences on $X \times X$ if 
for all $x^{\prime \prime}, x^{\prime}, y^{\prime \prime}, y^{\prime} \in X$ with $x^{\prime \prime}>x^{\prime}$ and $y^{\prime \prime}>y^{\prime}$,

$$
\pi\left(x^{\prime \prime}, y^{\prime \prime}\right)-\pi\left(x^{\prime}, y^{\prime \prime}\right) \leq(\geq) \pi\left(x^{\prime \prime}, y^{\prime}\right)-\pi\left(x^{\prime}, y^{\prime}\right)
$$

$\pi$ is a valuation if it has both decreasing and increasing differences. ${ }^{8}$

Definition 5 (Additively Separable) We say that a payoff function $\pi$ is additively separable if $\pi(x, y)=f(x)+g(y)$ for some functions $f, g: X \longrightarrow \mathbb{R}$.

Potential functions are often useful for obtaining results on convergence of learning algorithms to equilibrium, existence of pure equilibrium, and equilibrium selection. The following notion of potential games was introduced by Monderer and Shapley (1996).

Definition 6 (Exact potential games) The symmetric two-player game $(X, \pi)$ is an exact potential game if there exists an exact potential function $P: X \times X \longrightarrow \mathbb{R}$ such that for all $y \in X$ and all $x, x^{\prime} \in X,{ }^{9}$

$$
\begin{aligned}
& \pi(x, y)-\pi\left(x^{\prime}, y\right)=P(x, y)-P\left(x^{\prime}, y\right) \\
& \pi(x, y)-\pi\left(x^{\prime}, y\right)=P(y, x)-P\left(y, x^{\prime}\right) .
\end{aligned}
$$

Proposition 1 Let $(X, \pi)$ be an arbitrary symmetric two-player zero-sum game and $X$ be a totally ordered set. Then the following statements are equivalent:

(i) $\pi$ has decreasing differences on $X \times X$,

(ii) $\pi$ has increasing differences on $X \times X$,

(iii) $\pi$ is a valuation,

(iv) $\pi$ is additively separable,

(v) $(X, \pi)$ has an exact potential.

\footnotetext{
${ }^{8}$ For the two-dimensional case, increasing differences are equivalent to supermodularity, so the two terms can be used interchangeably (see Topkis, 1998, Chapter 2.6).

${ }^{9}$ Given the symmetry of $(X, \pi)$, the second equation plays the role usually played by the quantifier "for all players" in the definition of potential games.
} 
Proof. Let $X$ be a totally ordered set such that $\pi$ has decreasing differences on $X \times X$ if for all $x^{\prime \prime \prime}, x^{\prime \prime}, x^{\prime}, x \in X$ with $x^{\prime \prime \prime}>x^{\prime}$ and $x^{\prime \prime}>x$,

$$
\pi\left(x^{\prime \prime \prime}, x^{\prime \prime}\right)-\pi\left(x^{\prime}, x^{\prime \prime}\right) \leq \pi\left(x^{\prime \prime \prime}, x\right)-\pi\left(x^{\prime}, x\right)
$$

Since $(X, \pi)$ is a symmetric two-player zero-sum game, $\pi\left(x^{\prime}, x\right)=-\pi\left(x, x^{\prime}\right)$ for all $x, x^{\prime} \in$ $X$. Hence, we can rewrite this inequality as

$$
-\pi\left(x^{\prime \prime}, x^{\prime \prime \prime}\right)+\pi\left(x^{\prime \prime}, x^{\prime}\right) \leq-\pi\left(x, x^{\prime \prime \prime}\right)+\pi\left(x, x^{\prime}\right) .
$$

Rearranging inequality (1) yields the definition of increasing differences,

$$
\pi\left(x^{\prime \prime}, x^{\prime}\right)-\pi\left(x, x^{\prime}\right) \leq \pi\left(x^{\prime \prime}, x^{\prime \prime \prime}\right)-\pi\left(x, x^{\prime \prime \prime}\right)
$$

Hence (i) if and only if (ii). (iii) follows from the equivalence of (i) and (ii).

By Topkis (1998, Theorem 2.6.4.), a function $\pi(x, y)$ is additively separable on $X \times X$ if and only if $\pi(x, y)$ it is a valuation. Thus, (iii) if and only if (iv).

Brânzei, Mallozzi and Tijs (2003, Theorem 1) show that a zero-sum game is an exact potential game if and only if it is additively separable. Hence, (iv) if and only if (v).

Corollary 2 Let $(X, \pi)$ be a symmetric two-player zero-sum game for which $X$ is nonempty compact subset of a topological space and $\pi$ is upper semicontinuous. If $(X, \pi)$ satisfies any of the properties ( $i)$ to $(v)$ of Proposition 1, then a pure equilibrium exists.

Proof. Since $X$ is compact and $\pi$ is upper semicontinuous, any player's best response correspondence of $(X, \pi)$ is nonempty by Weierstrass' Theorem. Since $\pi$ is additively separable under any property (i) to (v) by Proposition 1, the best response correspondence is constant. Thus, a pure equilibrium of $(X, \pi)$ exists.

For the remainder of this section, we consider the relationships between the results. Corollary 2 is implied by Theorem 2 if finite games are considered.

Remark 2 Let $X$ be a totally ordered set. If the symmetric zero-sum game $(X, \pi)$ satisfies any of the properties (i) to ( $v$ ) of Proposition 1 , then $(X, \pi)$ is quasiconcave.

Proof. If property (iv) holds then there are some functions $f, g: X \longrightarrow \mathbb{R}$ such that $\pi(x, y)=f(x)+g(y)$ for all $x, y \in X$. Then $(X, \pi)$ is quasiconcave if and only if $f(x) \geq \min \left[f\left(x^{\prime}\right), f\left(x^{\prime \prime}\right)\right]$. Since $X$ is a totally ordered set, we can order it such that 
$x^{\prime} \geq x$ if and only if $f\left(x^{\prime}\right) \geq f(x)$. Thus, $(X, \pi)$ is quasiconcave.

The converse is not true as the following example shows.

Example 2 Consider a symmetric two-player $3 x 3$ zero-sum game. ${ }^{10}$

$$
\begin{aligned}
& A \\
& A \\
& B \\
& C
\end{aligned} \quad\left(\begin{array}{ccc}
0 & -1 & -3 \\
1 & 0 & -1 \\
3 & 1 & 0
\end{array}\right)
$$

This game is quasiconcave but its payoff function is not a valuation, i.e., payoff differences in own strategies are not constant in the opponent's strategies.

Theorem 2 and Corollary 2 overlap in the important case of $2 \times 2$ games. It is straightforward to check that every symmetric $2 \mathrm{x} 2$ zero-sum game is quasiconcave and satisfies all of the properties (i) to (v) of Proposition 1.

\section{Application to Relative Payoff Games}

Consider now more generally a symmetric two-player (not necessarily zero-sum) game $(X, \pi)$. When instead of the payoff function $\pi$ the relative payoffs are considered, then symmetric two-player games give naturally rise to the class of symmetric zero-sum games.

Definition 7 (Relative payoff game) Given a symmetric two-player game $(X, \pi)$, the associated relative payoff game is $(X, \Delta)$, where the relative payoff function $\Delta: X \times X \longrightarrow$ $\mathbb{R}$ is defined by

$$
\Delta(x, y)=\pi(x, y)-\pi(y, x)
$$

The relative payoff of a player is the difference between his payoff and the payoff of his opponent.

Remark 3 Every relative payoff game is a symmetric zero-sum game. Conversely, for every symmetric zero-sum game, there is a symmetric two-player game for which the relative payoff game is the symmetric zero-sum game.

\footnotetext{
${ }^{10}$ We thank an anonymous referee for suggesting this example.
} 
Proof. Note that by definition, $\Delta(x, y)=\pi(x, y)-\pi(y, x)=-[\pi(y, x)-\pi(x, y)]=$ $-\Delta(y, x)$ and hence $(X, \Delta)$ is a symmetric zero-sum game. For the converse, if $(X, \Delta)$ is a symmetric zero-sum game, then $(X, \pi)$ with $\pi(x, y)=\frac{1}{2} \Delta(x, y)$ is a symmetric two-player game for which $(X, \Delta)$ is the relative payoff game. To see this, note that since $(X, \Delta)$ is a symmetric zero-sum game, we must have that $\left(X, \frac{1}{2} \Delta\right)$ is a symmetric zero-sum game. Note further that $\Delta(x, y)=\pi(x, y)-\pi(y, x)=\frac{1}{2} \Delta(x, y)-\frac{1}{2} \Delta(y, x)=\frac{1}{2} \Delta(x, y)+\frac{1}{2} \Delta(x, y)$, where the last equality follows from the fact that $\left(X, \frac{1}{2} \Delta\right)$ is a symmetric zero-sum game.

The remark shows that every relative payoff game is a symmetric zero-sum game, and that relative payoff games do not impose any restriction on the class of symmetric zero-sum games. Every symmetric zero-sum game is a relative payoff game of some symmetric two-player game. Note also that different symmetric two-player games may have the same relative payoff game.

What outcomes in a symmetric two-player game correspond to pure equilibria in its associated relative payoff game? To answer this question we introduce the notion of finite population evolutionary stable strategy (Schaffer, 1988, 1989). This concept is appropriate when "playing the field", i.e. when players are matched against all other players except themselves.

Definition 8 (fESS) A strategy $x^{*} \in X$ is a finite population evolutionary stable strategy (fESS) of the game $(X, \pi)$ if

$$
\pi\left(x^{*}, x\right) \geq \pi\left(x, x^{*}\right) \text { for all } x \in X .
$$

In terms of the associated relative payoff game, inequality (2) is equivalent to

$$
\Delta\left(x^{*}, x\right) \geq 0 \text { for all } x \in X \text {. }
$$

Schaffer $(1988,1989)$ observed that $x^{*}$ is a fESS of the symmetric game $(X, \pi)$ if and only if $\left(x^{*}, x^{*}\right)$ is a pure Nash equilibrium of the relative payoff game $(X, \Delta) \cdot{ }^{11}$

Our results in Section 2 provide existence results for fESS of $(X, \pi)$ when conditions are imposed on the associated relative payoff game $(X, \Delta)$. That is, a symmetric game $(X, \pi)$ has a fESS if and only if its associated relative payoff game $(X, \Delta)$ is not a gRPS. In particular, every symmetric $2 \times 2$ game has a fESS. Furthermore, if the relative

${ }^{11}$ See Ania (2008) and Hehenkamp, et al. (2010) for further discussion. 
payoff function $\Delta$ associated to a finite game $(X, \pi)$ is quasiconcave, then a fESS exists. Finally, if the relative payoff game $(X, \Delta)$ associated to $(X, \pi)$ satisfies the properties of Corollary 2, then a fESS exists.

There is an interesting connection between symmetric two-player games and their relative payoff games with regard to the existence of an exact potential function.

Theorem 3 Let $(X, \pi)$ be a symmetric two-player game with the associated relative payoff games $(X, \Delta) .(X, \pi)$ is an exact potential game if and only if $(X, \Delta)$ is an exact potential game.

Proof. If $P$ is an exact potential function of a symmetric two-player game $(X, \pi)$, then $P$ is symmetric, i.e. $P(x, y)=P(y, x)$ for all $x, y \in X$. To see this note that $P$ being an exact potential $(X, \pi)$ implies for all $x, y \in X$

$$
\begin{aligned}
& \pi(x, y)-\pi(y, y)=P(x, y)-P(y, y) \text { and } \\
& \pi(x, y)-\pi(y, y)=P(y, x)-P(y, y)
\end{aligned}
$$

Hence $P(y, x)=P(x, y)$.

Adding some suitable additional terms on both sides that by symmetry of $P$ are equal we obtain for all $x, x^{\prime}, y, y^{\prime} \in X$

$$
\left(P\left(x^{\prime}, y\right)-P(x, y)\right)-\left(P\left(y, x^{\prime}\right)-P\left(y^{\prime}, x^{\prime}\right)\right)=\left(P\left(x^{\prime}, y^{\prime}\right)-P\left(x, y^{\prime}\right)\right)-\left(P(y, x)-P\left(y^{\prime}, x\right)\right) .
$$

Since $P$ is an exact potential function of $(X, \pi)$ we can rewrite this equation

$\left(\pi\left(x^{\prime}, y\right)-\pi(x, y)\right)-\left(\pi\left(y, x^{\prime}\right)-\pi\left(y^{\prime}, x^{\prime}\right)\right)=\left(\pi\left(x^{\prime}, y^{\prime}\right)-\pi\left(x, y^{\prime}\right)\right)-\left(\pi(y, x)-\pi\left(y^{\prime}, x\right)\right)$.

Rearranging terms yields

$\left(\pi\left(x^{\prime}, y\right)-\pi\left(y, x^{\prime}\right)\right)-(\pi(x, y)-\pi(y, x))=\left(\pi\left(x^{\prime}, y^{\prime}\right)-\pi\left(y^{\prime}, x^{\prime}\right)\right)-\left(\pi\left(x, y^{\prime}\right)-\pi\left(y^{\prime}, x\right)\right)$

Using the relative payoff function $\Delta$, we obtain

$$
\Delta\left(x^{\prime}, y\right)-\Delta(x, y)=\Delta\left(x^{\prime}, y^{\prime}\right)-\Delta\left(x, y^{\prime}\right) .
$$

Thus $\Delta$ is a valuation which by Proposition 1 is equivalent to $(X, \Delta)$ being an exact potential game.

Often it is rather difficult to verify the existence of an exact potential function. Theorem 3 and Proposition 1 show that it is straight forward for symmetric two-player games. 
It is easy to verify whether the relative payoff function associated with the symmetric two-player game is a valuation.

Finally, the following two corollaries provide sufficient conditions imposed on the payoff function $\pi$ of the underlying game $(X, \pi)$ for the existence of a fESS.

Corollary 3 Consider a symmetric two-player game $(X, \pi)$ with a compact strategy set $X$ and a continuous payoff function. If $(X, \pi)$ is an exact potential game, then a fESS exists.

The corollary follows since by Theorem 3 the relative payoff game is also an exact potential game. Hence, Corollary 2 implies the existence of a pure equilibrium of $(X, \Delta)$, which is a fESS of $(X, \pi)$.

Corollary 4 Consider a symmetric two-player game $(X, \pi)$ with a compact strategy set $X$ and a payoff function that can be written as $\pi(x, y)=f(x)+g(y)+a(x, y)$ for some continuous functions $f, g: X \longrightarrow \mathbb{R}$ and a symmetric function $a: X \times X \longrightarrow \mathbb{R}$ (i.e., $a(x, y)=a(y, x)$ for all $x, y \in X)$. Then $(X, \pi)$ has a fESS.

The corollary follows since $\pi(x, y)=f(x)+g(y)+a(x, y)$ implies that in the relative payoff game the term $a(x, y)$ drops out, $\Delta(x, y)=f(x)-g(y)-f(y)+g(x)$. Again, Corollary 2 implies the existence of a fESS of $(X, \pi)$.

While at first glance the condition on payoffs in the last corollary looks restrictive, it is satisfied in many well-known textbook examples of two-player games including linear Cournot duopoly, versions of Bertrand competition, public goods games, common pool resource games, minimum effort coordination games, synergistic relationships, Diamond's search, Nash demand game, and Tullock rent seeking games (for details see Duersch, Oechssler, and Schipper, 2010). In applications, the function $a$ is often symmetric because it represents just the sum or the product of players' strategies.

\section{References}

[1] Alós-Ferrer, C. and A.B. Ania (2005). The evolutionary stability of perfectly competitive behavior, Economic Theory 26, 497-516.

[2] Ania, A. (2008). Evolutionary stability and Nash equilibrium in finite populations, with an application to price competition, Journal of Economic Behavior and Organization 65, 472-488. 
[3] Brânzei, R., Mallozzi, L., and S. Tijs (2003). Supermodular games and potential games, Journal of Mathematical Economics 39, 39-49.

[4] Duersch, P., Oechssler, J., and B.C. Schipper (2010). Unbeatable imitation, mimeo., University of Heidelberg and the University of California, Davis.

[5] Hehenkamp, B., Possajennikov, A., and T. Guse (2010). On the equivalence of Nash and evolutionary equilibrium in finite populations, Journal of Economic Behavior and Organization 73, 254-258.

[6] Leininger, W. (2006). Fending off one means fending off all: evolutionary stability in quasi-submodular games, Economic Theory 29, 713-719.

[7] Matros, A., Temzelides, T., and J. Duffy (2009). Competitive behavior in market games: Evidence and theory, mimeo.

[8] Monderer, D. and L.S. Shapley (1996). Potential games, Games and Economic Behavior 14, 124-143.

[9] Nash, J. (1951). Non-cooperative games, Annals of Mathematics 54, 286-295.

[10] von Neumann, J. (1928). Zur Theorie der Gesellschaftsspiele, Mathematische Annalen 100, 295-320.

[11] Nydegger, R.V. and G. Owen (1974). Two-person bargaining: An experimental test of the Nash axioms, International Journal of Game Theory 3, 239-249.

[12] Possajennikov, A. (2003). Evolutionary foundation of aggregative-taking behavior, Economic Theory 21, 921-928.

[13] Radzik, T. (1991). Saddle point theorems, International Journal of Game Theory 20, 23-32.

[14] Roth, A.E. and M.W.K. Malouf (1979). Game-theoretic models and the role of information in bargaining, Psychological Review 86, 574-594.

[15] Schaffer, M.E. (1989). Are profit-maximizers the best survivors?, Journal of Economic Behavior and Organization 12, 29-45.

[16] Schaffer, M.E. (1988). Evolutionary stable strategies for a finite population and a variable contest size, Journal of Theoretical Biology 132, 469-478. 
[17] Shapley, L.S. (1964). Some topics in two-person games, in: Dresher, M., Shapley, L.S. and A.W. Tucker (eds.), Advances in Game Theory, Annals of Mathematical Studies 52, 1-28.

[18] Tanaka, Y. (2000). A finite population ESS and a long run equilibrium in an nplayers coordination game, Mathematical Social Sciences 39, 195-206.

[19] Topkis, D. M. (1998). Supermodularity and complementarity, Princeton, New Jersey: Princeton University Press.

[20] Vega-Redondo, F. (1997). The evolution of Walrasian behavior, Econometrica 65, 375-384. 